\title{
Alive and kicking: microRNAs in mammalian antiviral responses
}

\begin{abstract}
A new report published in Nature provides some of the first evidence that changes in cellular microRNA (miRNA) levels are a component of the mammalian innate immune response to viruses. Michael David and colleagues show that, in mammals, type I interferons (IFN $\alpha$ and IFN $\beta$ ) upregulate several cellular miRNAs that can inhibit the replication of, or infection by, hepatitis $C$ virus HCV.

The targeting of viral RNAs by RNA interference (RNAi), which is mediated by non-coding miRNAs, is an important antiviral defence mechanism that is used by both plants and invertebrates, but there has been no evidence that cellular miRNAs have a similar role in mammals. Instead, it has been proposed that IFNs are the key antiviral defence mechanisms in mammalian cells. But, could type I IFNs use RNAi as part of their antiviral mechanism?

Using microarray technology, David and colleagues showed that IFN $\beta$ rapidly modulates the expression of many cellular miRNAs, eight of which matched specific target sequences within the HCV RNA genome. Interestingly, no matches
\end{abstract}

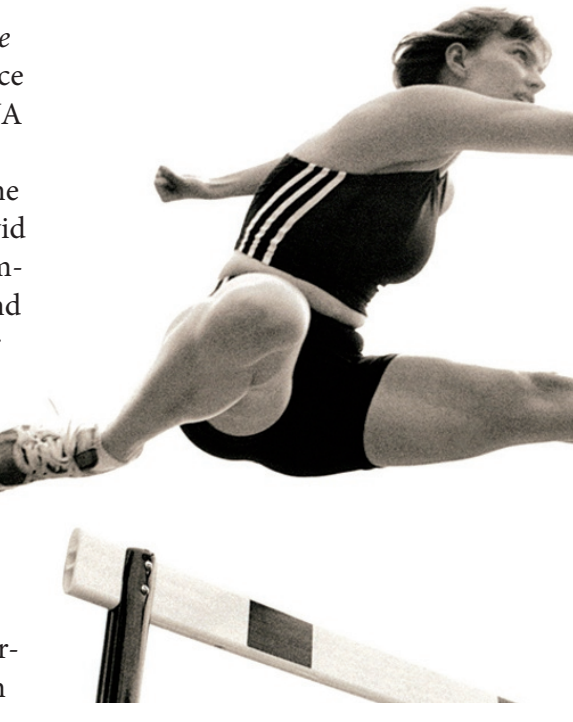

were found in a similar analysis that used hepatitis B virus, which is a DNA virus.

Could these eight IFN $\beta$-induced miRNAs inhibit HCV replication? Synthetic mimics of the miRNAs were introduced into cells from the Huh7 human hepatoma cell line that harboured an HCV replicon and the antiviral effects of IFN $\beta$ were reproduced, which substantially attenuated viral replication. At least two of the eight miRNAs were shown to directly target $\mathrm{HCV}$ genomic RNA. The authors next investigated whether the modulation of cellular miRNA levels was necessary for IFN $\beta$ to prevent HCV replication. This was indeed the case, as neutralization of the miRNAs reduced the antiviral effects of IFN $\beta$ against HCV. In addition, the authors showed that expression of miR-122, which is expressed in the liver and has been shown to be essential for $\mathrm{HCV}$ replication, is downregulated in response to IFN $\beta$.

This paper, therefore, indicates that, similar to plants and invertebrates, cellular miRNAs in mammals are modulated by the IFN system to fight viral infections.

ORIGINAL RESEARCH PAPER Pedersen, I. M. et al. Interferon modulation of cellular microRNAs as an antiviral mechanism. Nature 449, 919-922 (2007) 